\title{
Neurovascular disorders in children: an updated practical guide
}

\author{
Richa Sinha ${ }^{1}$, Saipriya Ramji ${ }^{2}$ \\ ${ }^{1}$ Department of Neuroradiology, Royal Preston Hospital, Preston, UK; ${ }^{2}$ Department of Neuroradiology, Charing Cross Hospital, London, UK \\ Contributions: (I) Conception and design: All authors; (II) Administrative support: All authors; (III) Provision of study materials or patients: All \\ authors; (IV) Collection and assembly of data: All authors; (V) Data analysis and interpretation: All authors; (VI) Manuscript writing: All authors; (VII) \\ Final approval of manuscript: All authors. \\ Correspondence to: Dr. Richa Sinha. Neuroradiology Fellow, Department of Neuroradiology, Royal Preston Hospital, Sharoe Green Lane, Preston, \\ PR2 9HT, UK. Email: Richasinha@doctors.org.uk.
}

\begin{abstract}
Neurovascular disorders are an important cause of brain injury in the paediatric population, leading to substantial mortality and morbidity. These include ischaemic, haemorrhagic strokes, and cerebral venous sinus thromboses. Despite remarkable improvements in our understanding and management of strokes in general, and in the quality of immediate care and rehabilitation, the outcomes in childhood strokes lag far behind adult strokes. This is a multifaceted problem. There remain several challenges including delays in recognition due to vague clinical presentations, a failure to consider strokes in many cases, delays in obtaining imaging and inadequate imaging when performed, as well as limited evidence and availability for hyper acute intervention in children. Stroke in children is also a vastly different entity to adult strokes, with a unique set of risk factors and often with an underlying cause to be found. This review is by no means exhaustive, but instead aims to provide an overview of the epidemiology, sub-classification and aetiology of paediatric neurovascular disorders, mainly ischaemic, haemorrhagic strokes and cerebral venous sinus thrombosis. We also focus on the risk factors for each of these, the clinical presentation, recommended diagnostic protocol, pertinent imaging findings, as well as the current treatment and follow-up practices.
\end{abstract}

Keywords: Arterial ischaemic stroke (AIS); arteriovenous malformation (AVM); cerebral venous sinus thrombosis (CVST); haemorrhagic stroke

Submitted Jul 22, 2020. Accepted for publication Dec 24, 2020.

doi: $10.21037 / \mathrm{tp}-20-205$

View this article at: http://dx.doi.org/10.21037/tp-20-205

\section{Classification of paediatric stroke}

Stroke is defined by the WHO as a rapid deterioration in neurological symptoms lasting more than 24 hours with a vascular origin (1). It is an important cause of longterm morbidity and mortality in children. The incidence of childhood stroke varies amongst different population studies and lies between 2-13 per 100,000 (2-4).

Paediatric stroke is classified as either perinatal $(\leq 28$ days of age) or childhood ( $>28$ days of age) (5). The focus of this article will be on childhood stroke.

Childhood stroke can be further subdivided into ischaemic and haemorrhagic stroke (Figure 1). Ischaemic stroke encompasses both arterial ischaemic stroke (AIS) and venous infarction secondary to cerebral venous sinus thrombosis (CVST) or cortical vein thrombosis (6). CVST is frequently accompanied by areas of parenchymal haemorrhage.

\section{AIS}

\section{Epidemiology and risk factors}

Paediatric stroke is classified as either perinatal/neonatal (28 days of age) or childhood ( $>28$ days of age). The definition of AIS is shown in Table 1.

The major risk factors that cause adult strokes such as atherosclerosis and associated conditions such as hypertension and dyslipidaemia are irrelevant in childhood. The aetiology of AIS in childhood differs from adults, is complex and more often multifactorial. The age of the child is also an important factor with stroke in the neonatal stage having a unique set of considerations to strokes in an older 


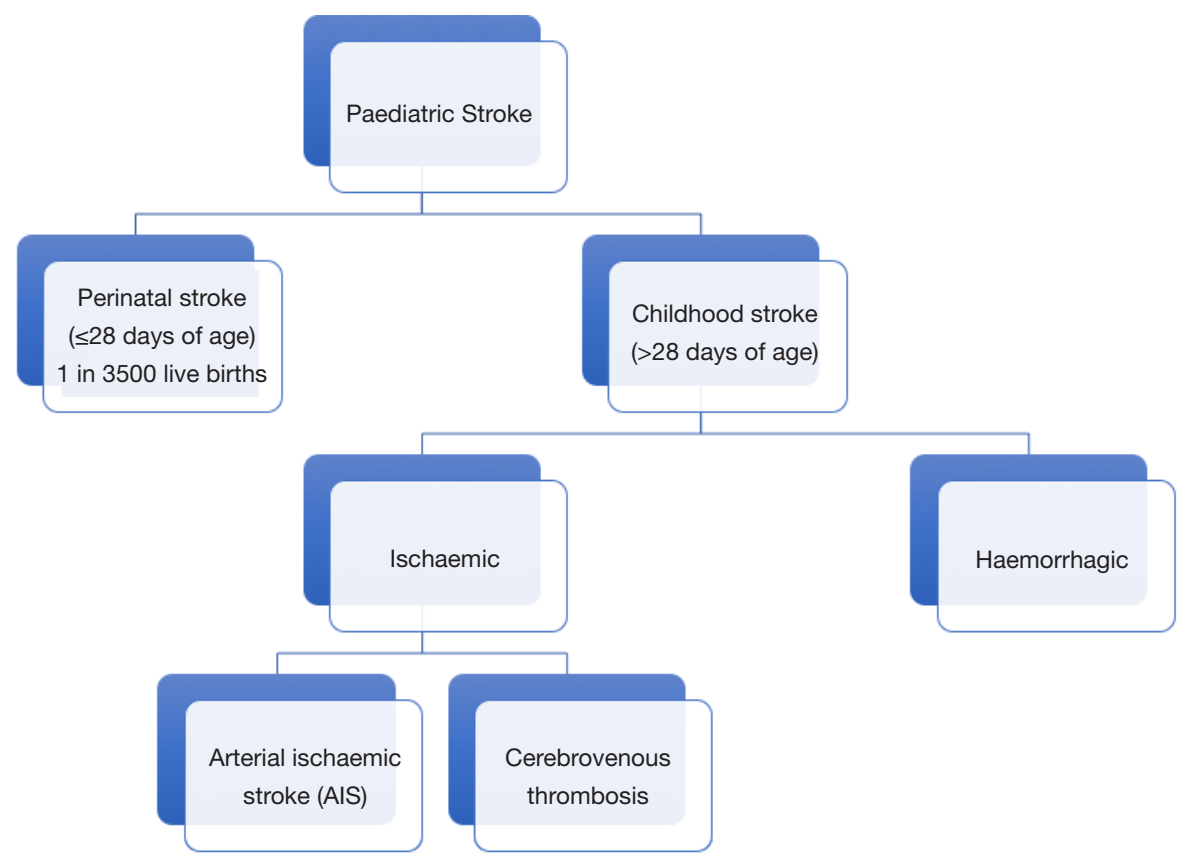

Figure 1 Classification of paediatric stroke.

Table 1 Definition of AIS (7)

Definition of AIS
Acute focal neurological deficit consistent with arterial
ischaemia
And Ped NIHSS $\geq 4$ and $\leq 24$
And CT has excluded intracranial haemorrhage
Or MR shows evidence of acute ischaemia

AIS, arterial ischaemic stroke; Ped NIHSS, Paediatric National Institute of Health Stroke scale.

child. The age of the child, beyond the neonatal stage, can also be of some limited use in narrowing down the causes, with genetic or metabolic causes more likely to present in younger children. As the child grows, acquired conditions such as post viral arteriopathies tend to feature more prominently.

Many different approaches to classifying these risk factors and causes have been described in the literature. One such classification from the paediatric stroke working group 2017 has been summarised in Table 2 (7).

The most common cause in children with AIS is a cerebral arteriopathy found in $50-80 \%$ of the cases $(6,8,9)$. Arteriopathy is also the single most important predictor of recurrence. The diagnoses causing arteriopathy include focal cerebral arteriopathy of childhood, moyamoya disease, arterial dissection and central nervous system (CNS) vasculitis. These will therefore be discussed in more detail below.

\section{Clinical presentation}

The clinical presentation of strokes in children is much more varied than adult strokes. This, coupled with prevalent misconceptions in the medical community, leads to failure to diagnose in time, and inappropriate management.

Mallick et al. [2014], outlined the myriad of clinical presentations and focal neurological features in particular hemiparesis was seen in $72 \%$ of cases (10). Clinicians must recognise the wide range of clinical signs and symptoms these patients present with and be able to facilitate the recommended diagnostic protocol (Table 4).

\section{Diagnostic workup}

\section{Imaging protocol}

Urgent brain imaging should be carried out in children who present with acute focal neurological deficits, aphasia or reduced level of consciousness. In addition, urgent brain imaging should also be considered when a child presents with a number of other presentations outlined in Table 4. 
Table 2 Risk factors for stroke in the paediatric population (7)

\begin{tabular}{|c|c|}
\hline Risk category & Risk factors/diagnoses \\
\hline \multirow[t]{6}{*}{ Arteriopathy } & Transient cerebral arteriopathy \\
\hline & Varicella zoster infection most common \\
\hline & Moyamoya \\
\hline & Arterial dissection \\
\hline & CNS vasculitis \\
\hline & Sickle cell disease (SCD) \\
\hline \multirow{4}{*}{$\begin{array}{l}\text { Congenital cardiac } \\
\text { disease }\end{array}$} & Right to left shunt \\
\hline & Lipoprotein(a) \\
\hline & anticardiolipin antibody (ACLA) \\
\hline & combined prothrombotic disorders \\
\hline \multicolumn{2}{|c|}{ Cardiac surgery/intervention } \\
\hline \multirow[t]{2}{*}{ Gender/ethnicity } & Black or Asian ethnicity \\
\hline & Male gender \\
\hline \multirow[t]{12}{*}{ Genetic risk factors } & Thrombophilia \\
\hline & $\begin{array}{l}\text { Genetic: factor V Leiden, PT20210, } \\
\text { MTHFRc677T, protein C deficiency, } \\
\text { increased lipoprotein(a) }\end{array}$ \\
\hline & Acquired: antiphospholipid syndrome \\
\hline & Metabolic disorders \\
\hline & Organic acidurias \\
\hline & Mitochondrial disorders \\
\hline & Lysosomal disorders \\
\hline & Neurofibromatosis type 1 \\
\hline & Tuberous sclerosis \\
\hline & Fibromuscular dysplasia \\
\hline & PHACES syndrome \\
\hline & $\begin{array}{l}\text { Specific genetic mutations: described in } \\
\text { Table } 3\end{array}$ \\
\hline \multirow[t]{6}{*}{ Miscellaneous } & Iron deficiency anaemia \\
\hline & Radiotherapy \\
\hline & High alpha antitrypsin \\
\hline & Trauma \\
\hline & Under-vaccination \\
\hline & Multiple risk factors \\
\hline
\end{tabular}

Diagnostic delays are more common in ischaemic than haemorrhagic stroke with a median time of symptom onset to neuroimaging at 24 hours in ischaemic compared to 3 hours in haemorrhagic stroke $(5,11)$. Although this disparity is felt to be multifactorial, some of the key problems lie with delay in appropriate and adequate imaging studies as well as the low sensitivity of CT for acute cerebral infarction (11).

Neuroimaging is crucial to differentiate between true cerebral infarction and haemorrhage as well as the myriad of stroke mimics. Ladner et al. 2015, conducted a prospective observational study of 124 children in whom a stroke protocol was activated (12). Of these, only $26 \%$ had a final diagnosis of stroke or transient ischaemic attack, emphasising the prevalence of stroke mimics (12).

The most common stroke mimics included migraine (17\%), seizure (15\%), neurological emergencies including meningitis/encephalitis and neoplasm (14\%) (12). These can be distinguished on imaging and therefore early imaging is imperative in the evaluation of suspected paediatric stroke. In patients with migraine, MR may be normal or reveal small non-specific white matter hyperintensities which are subcortical in location. MR imaging may be particularly helpful in hemiplegic migraine, a rare variant of migraine. In these cases, MR may demonstrate cortical swelling, venous dilatation on susceptibility weighted imaging (SWI) in the contralateral hemisphere and gyriform enhancement. The absence of diffusion restriction and the pattern of cortical swelling not conforming to a vascular territory in combination with a characteristic clinical history helps to differentiate hemiplegic migraine from AIS.

Meningitis and encephalitis have distinct imaging features which help distinguish them from AIS including meningeal enhancement which is usually diffuse. Diffusion restriction may be present in encephalitis and seizure related change, however, this would not conform to a vascular territory and is predominantly cortical in location.

In an acute setting, a non-contrast CT head with CT angiogram (CTA) should be the first radiological investigation to be carried out, within 1 hour of symptom onset and reviewed immediately. The CTA should be from arch to vertex unless there is an intracranial haemorrhage in which case, a limited angiogram of the Circle of Willis is sufficient (7).

If MR imaging is available within 1 hour of presentation, then this is the gold standard modality of choice and is 
Table 3 A summary of various metabolic and genetic conditions associated with strokes and associated imaging findings

\begin{tabular}{|c|c|}
\hline Risk category/conditions & Associated important clinical and imaging findings \\
\hline \multirow[t]{2}{*}{ Coagulopathies } & Disorders of Protein C, S, plasminogen, anti-thrombic, fibrinogen deficiency and anti cardiolipin antibodies \\
\hline & Polycythemia, anemia and platelet disorders are also risk factors \\
\hline \multirow{2}{*}{$\begin{array}{l}\text { Homocystenuria and } \\
\text { homocystenemia }\end{array}$} & Rarely can be genetic in nature, and can be associated with heritable ectopia lentis \\
\hline & High homocysteine levels is a risk factor of cardiovascular disease and thrombosis \\
\hline \multirow{2}{*}{$\begin{array}{l}\text { MELAS (mitochondrial } \\
\text { myopathy, encephalopathy, } \\
\text { lactic acidosis and stroke-like } \\
\text { episodes) }\end{array}$} & Infarcts of differing ages 'shifting pattern spread' \\
\hline & Increased diffusivity on DWI sequences, indicating a mix of vasogenic and cytotoxic oedema \\
\hline ACTA2 mutation (Figure 2) & $\begin{array}{l}\text { An occlusive vasculopathy with distinct clinical and angiographic findings. Chronically fixed dilated pupils is } \\
\text { a characteristic clinical marker }\end{array}$ \\
\hline \multirow[t]{4}{*}{ Notch 3 mutation (CADASIL) } & An autosomal dominant microvasculopathy \\
\hline & $\begin{array}{l}\text { Mainly leads to small vessel involvement but can also lead to aortic disease, coronary artery disease, stroke, } \\
\text { moyamoya disease and multisystemic smooth muscle dysfunction }\end{array}$ \\
\hline & $\begin{array}{l}\text { On MR imaging, extensive white matter T2W hyperintensity, with classical anterior temporal lobe and } \\
\text { external capsular predilection }\end{array}$ \\
\hline & $\begin{array}{l}\text { The autosomal recessive counterpart CARASIL has a milder outlook with stroke-like episodes usually from } \\
\text { the } 3^{\text {rd }} \text { decade }\end{array}$ \\
\hline \multirow{2}{*}{$\begin{array}{l}\text { ABCC6 mutation } \\
\text { (Pseudoxanthoma elasticum) }\end{array}$} & Autosomal recessive mutation \\
\hline & Often affects skin first with papular lesions and skin laxity \\
\hline $\begin{array}{l}\text { JAG1 mutation (Alagille } \\
\text { syndrome) }\end{array}$ & Notch 2 mutation has also been reported in Alagille syndrome \\
\hline \multirow{3}{*}{$\begin{array}{l}\text { ATP7A mutation (Menke's } \\
\text { disease/trichopoliodystrophy) }\end{array}$} & An X-linked recessive disorder involving genes coding for copper transport \\
\hline & Sparse and friable hair is a unique feature, hence is also termed 'kinky-hair syndrome' \\
\hline & $\begin{array}{l}\text { On neuroimaging, tortuous intracranial vessels, extra-axial collections, and a predilection for strokes (both } \\
\text { ischaemic and haemorrhagic have been described }\end{array}$ \\
\hline \multirow{2}{*}{$\begin{array}{l}\text { SLC2A10 mutation (arterial } \\
\text { tortuosity syndrome) }\end{array}$} & An autosomal recessive disorder which leads to occlusive arteriopathy \\
\hline & Tortuosity of the aorta and large vessels is also described \\
\hline \multirow{2}{*}{$\begin{array}{l}\text { GLA mutation } \\
\text { (Fabry's disease) }\end{array}$} & An X linked lysosomal disorder which leads to small vessel ischaemia, strokes, and multi-infarct dementia \\
\hline & Basilar artery dolichoectasia is also a characteristic imaging finding \\
\hline \multirow{2}{*}{$\begin{array}{l}\text { ELN gene mutation (Williams- } \\
\text { Bueren syndrome) }\end{array}$} & Most patients have supravalvular aortic stenosis \\
\hline & Some children may also have occlusive cerebrovascular disease \\
\hline \multirow[t]{2}{*}{$\begin{array}{l}\text { NF1 gene mutation } \\
\text { (Neurofibromatosis type 1) }\end{array}$} & $\begin{array}{l}\text { Excessive smooth muscle cell proliferation and vascular occlusion. Imaging may reveal diffuse cerebral } \\
\text { arteriopathy with occlusions and aneurysms }\end{array}$ \\
\hline & Associated with moyamoya syndrome \\
\hline
\end{tabular}



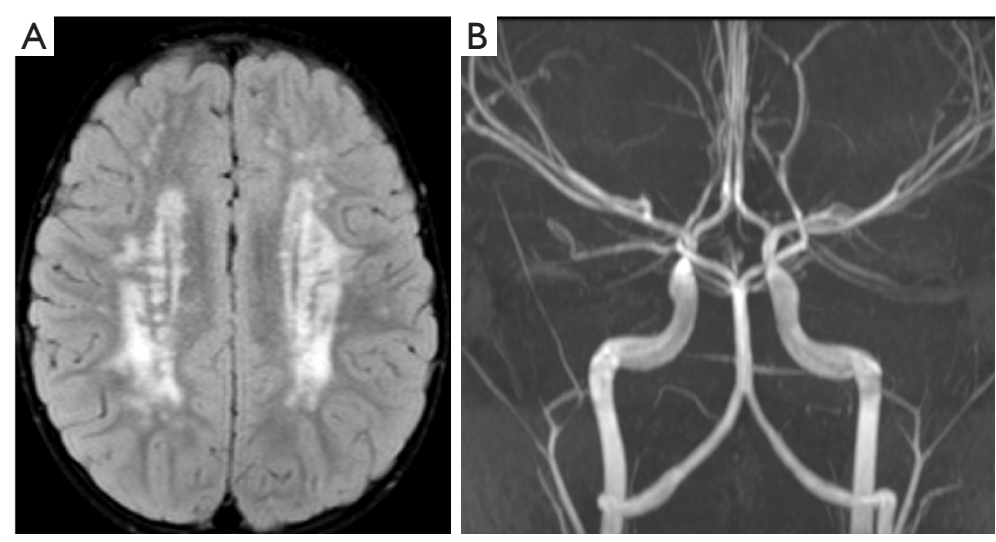

Figure 2 ACTA2 mutation. MR imaging in a 6-year-old girl with a history of stroke and TIAs. Axial FLAIR image (A) demonstrates numerous, deep watershed and periventricular infarcts. MR TOF imaging (B) shows a characteristic dilatation of the proximal ICAs while the terminal ICAs are severely thinned and 'straightened' with attenuation of the peripheral vasculature. FLAIR, fluid attenuated inversion recovery; TOF, time of flight.

Table 4 Variable clinical presentations associated with strokes in children. Adapted from (7)

\begin{tabular}{l} 
Stroke should be considered if a child presents with any of the \\
following symptoms \\
\hline New onset focal seizure \\
New onset severe headache \\
Unexplained altered mental status \\
New onset ataxia, vertigo or dizziness \\
Sudden onset neck pain or neck stiffness \\
Witnessed acute focal neurological deficit which has resolved \\
The presence of fever, nausea vomiting or change in behaviour \\
in children does not exclude stroke
\end{tabular}

therefore preferable. CT imaging has limited sensitivity and fails to identify the cause of patient's symptoms (ischaemic stroke or stroke mimics) in $34-40 \%$ of cases $(11,13)$. A negative CT head can be falsely reassuring and delay the request for MR brain. Many centres may also struggle to offer MR imaging on an acute basis and in such cases, if the initial CT is negative, the MR should be performed within 24 hours of presentation. The delay in neuroimaging also relate to other factors including a delay in consideration of clinical diagnosis of stroke, the need for sedation or general anaesthesia. A suggested imaging protocol is outlined in Table 5. MR imaging findings in paediatric infarcts are similar to that in adults with presence of restricted diffusion
Table 5 Imaging protocol for MRI in paediatric patients with suspected stroke

\begin{tabular}{l}
\hline Imaging protocol for MRI in patients with suspected stroke \\
\hline Axial T2WI \\
Axial T1WI \\
Axial +/- coronal FLAIR \\
Diffusion weighted imaging (DWI) \\
Susceptibility weighted imaging/Gradient echo (SWI/GRE) \\
Vascular imaging-TOF MR angiogram of intracranial +/- neck \\
vasculature \\
Vessel wall imaging can be considered if there is a strong \\
suspicion of vasculitis \\
\hline FLAIR, fluid attenuated inversion recovery; TOF, time of flight.
\end{tabular}

and corresponding T2W/FLAIR signal abnormality. Of note, diffusion restriction persists for a shorter period of time and pseudo-normalisation of the ADC map occur sooner in children.

Assessment of AIS onset time is crucial for determining whether a child can be treated with tissue plasminogen activator (tPA) or thrombectomy. Diffusion and FLAIR mismatch can be useful in the determination of the time window for intervention. Diffusion weighted imaging (DWI) is reliable at predicting the core infarct area and the utilisation of advanced neuroimaging techniques including perfusion imaging may help determine the penumbra. Although dynamic susceptibility contrast (DSC) perfusion 
Table 6 Investigations recommended in patients with confirmed AIS. Adapted from (7)

\begin{tabular}{ll}
\hline Investigation category & Tests \\
\hline Haematological & $\begin{array}{l}\text { Full blood count, iron status and } \\
\text { haemoglobinopathy screen }\end{array}$ \\
Biochemical & $\begin{array}{l}\text { Total plasma homocysteine, alpha } \\
\text { galactosidase, fasting blood sugar, } \\
\text { fasting cholesterol, and lipoprotein(a). } \\
\text { Lupus anticoagulant and ACLA, } \\
\text { and discuss beta 2GP1 testing with } \\
\text { haematology if necessary } \\
\text { Electrocardiogram (ECG) and } \\
\text { echocardiogram } \\
\text { Arch to vertex-CTA or MRA. Transcranial } \\
\text { Derebrovascular } \\
\text { Denetic }\end{array} \quad \begin{array}{l}\text { To be considered on a case by case } \\
\text { assessment }\end{array}$ \\
\hline
\end{tabular}

AIS, arterial ischaemic stroke; ACLA, anticardiolipin antibody; CTA, CT angiogram; MRA, MR angiogram; SCD, sickle cell disease.

imaging has been widely used in adults, it is unfavourable in children due to the administration of contrast medium.

Arterial spin labelling (ASL) is a non-invasive MR perfusion technique which can be obtained without the need for contrast administration and provides quantitative measurements of cerebral blood flow. Chen et al. 2009 suggests that ASL is able to demonstrate areas of hypoperfusion which correlate well with the infarct volume on follow up T2W images (14). In addition, ASL perfusion imaging may also provide useful information regarding collateral circulation (14). However, several logistical issues have been highlighted in literature including lack of standardisation of acquisition parameters and image processing within the paediatric cohort (15). In addition, the effects of sedation or general anaesthesia on cerebral perfusion has not been extensively investigated and therefore remains an important consideration in paediatric patients who undergo ASL imaging (15).

Vessel wall imaging is another promising technique and can provide information on vessel wall thickening and enhancement which may aid the diagnosis of arteriopathy and help to differentiate between aetiologies. The aim of vessel wall imaging is to suppress the luminal blood and CSF signal allowing visualisation of the vessel wall (16). The technique is based on 3T high resolution MR using black blood T1W sequences with fat suppression pre and post contrast administration aiming to suppress the blood and CSF allowing visualisation of the vessel wall. Vessel wall imaging can be considered to differentiate between subtypes of arteriopathies $(17,18)$.

\section{Other investigations}

Once the diagnosis of AIS has been confirmed, it is crucial to investigate for underlying risk factors. A recommended set of investigations as per the Royal college of Paediatrics and Child Health (RCPCH) guidelines 2017 have been summarised in Table 6.

\section{Background and imaging of specific conditions}

\section{Cerebral arteriopathy}

Cerebral arteriopathy is implicated in approximately $53 \%$ of all AIS cases (8). Two main forms of cerebral arteriopathy have been described and these are acute and chronic arteriopathies. Acute arteriopathy is most commonly triggered by infection or trauma in otherwise healthy children. Chronic arteriopathy in children is usually associated with underlying genetic disorders or systemic illnesses. The most common causes of cerebral arteriopathy include transient cerebral arteriopathy (TCA) $(25 \%)$, moyamoya disease (22\%), sickle cell disease $(8 \%)$, arterial dissection (20\%) and CNS vasculitis (12\%) (8). These are described in more detail below.

\section{TCA}

TCA is commonly associated with basal ganglia strokes and is characteristically focal and monophasic. The most common infection associated with arteriopathy is varicella zoster infection, thus being referred to as a post-varicella arteriopathy (19). Other viral infections such as parvovirus, cytomegalovirus, borrelia, enterovirus and helicobacter have also been implicated (19).

TCA is a provisional diagnosis, based on the initial imaging study, and is not a pathological entity. Follow up imaging is required to distinguish this from more chronic processes. This can be differentiated from moyamoya disease based on its non-progressive nature/regression, unilateral location, and lack of leptomeningeal collaterals. Another condition which overlaps with the arteriopathy syndromes is fibromuscular dysplasia, however, this is not common in childhood.

TCA most commonly involves the proximal middle cerebral artery but can also involve the distal internal carotid and proximal anterior cerebral arteries. The varicella zoster virus is thought to be harboured in the trigeminal ganglion and migrates through the trigeminal nerves innervating 

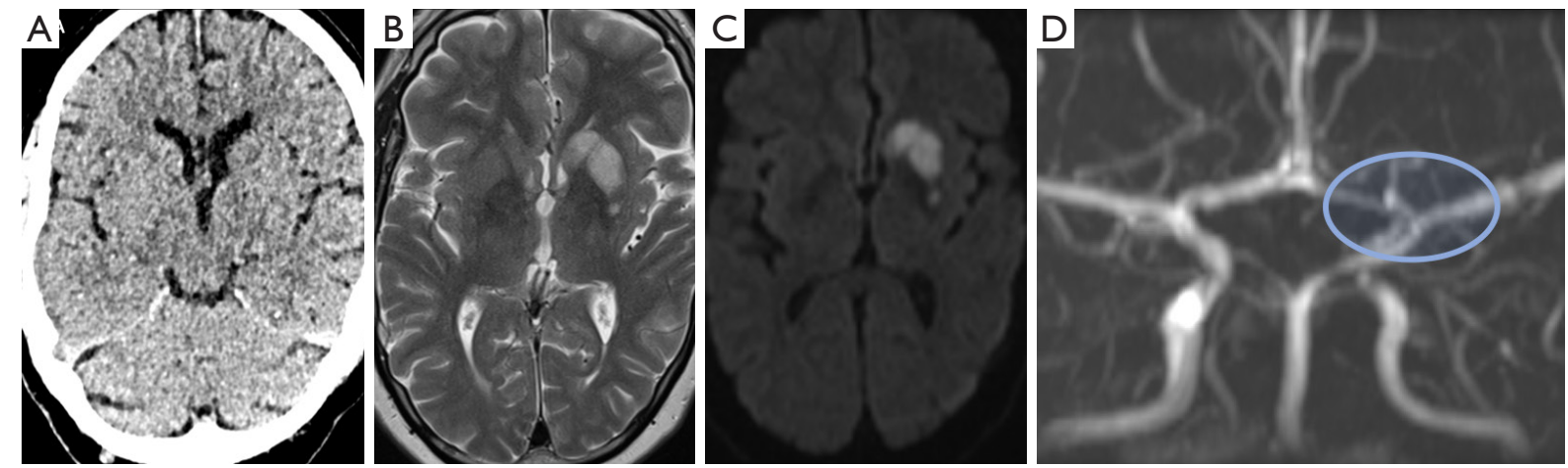

Figure 3 Transient cerebral arteriopathy. Axial CT (A) demonstrates very subtle low density within the left caudate head. Follow up MR with axial T2WI (B) and B1000 images (C) demonstrate acute infarction within the left caudate head and lentiform nucleus. Time of flight (TOF) imaging (D) confirms narrowing of the terminal left ICA, M1 and A1 (blue circle). There was complete resolution on follow up TOF imaging (not shown).
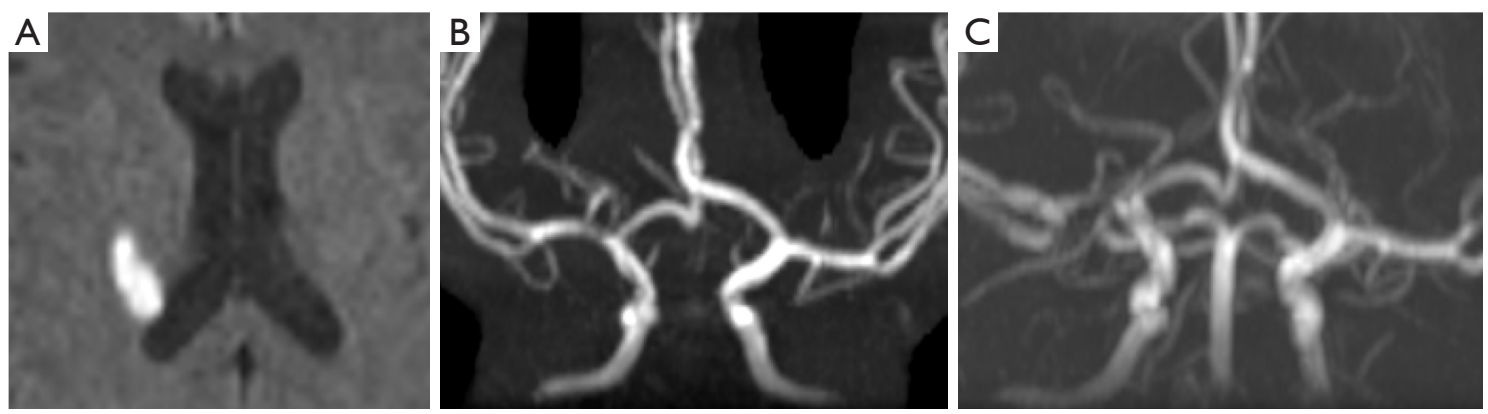

Figure 4 Chronic cerebral arteriopathy in a 4-year-old girl that presented with acute left sided hemiparesis. MR imaging demonstrate an acute infarct involving the right posterior limb of the internal capsule (A, DWI B1000). MRA TOF (B) reveals a focal narrowing of the right proximal MCA and ACA. Follow up MRA imaging demonstrates persistent narrowing of the right M1 and A1 segments (C). DWI, diffusion weighted imaging; MRA, MR angiogram; TOF, time of flight; MCA, middle cerebral artery; ACA, anterior cerebral artery.

the arterial tree at the level of the distal internal carotid artery (ICA) and proximal middle cerebral artery (MCA) causing inflammation within the arterial wall (20). On angiography, multifocal irregularity and reduction in calibre of the affected vessel can be seen (Figure 3). The condition usually either stabilises or improves in 3-6 months. If there is a progressive worsening in condition, a chronic arteriopathy or alternate diagnoses should be considered (Figure 4).

\section{Moyamoya disease/syndrome}

Moyamoya disease was named after the characteristic appearance of the dilated lenticulostriate perforating vessels on angiography described as 'a hazy puff of smoke'. When idiopathic, it is referred to as moyamoya disease and when associated with a systemic condition, moyamoya syndrome.

Moyamoya disease is a non-inflammatory, non- atherosclerotic progressive vasculopathy which involves the supraclinoid and terminal segments of the ICAs and the Circle of Willis (Figure 5). Involvement of the posterior circulation is also documented, although less common.

Common associations with moyamoya syndrome include Down's syndrome, sickle cell disease and Neurofibromatosis type 1. RNF213 gene mutation has been implicated in $56 \%$ of Asian American patients with moyamoya disease although the prevalence of this mutation is much lower in the non-Asian population (6).

On MR T2W sequences, multiple flow voids may be seen in the region of the basal ganglia and suprasellar cistern relating to collaterals. Bright sulci, also referred to as the "ivy sign" may be seen on FLAIR. MR angiogram (MRA) demonstrates circumferential luminal narrowing. Secondary aneurysms are also possible. Cerebral perfusion imaging, 

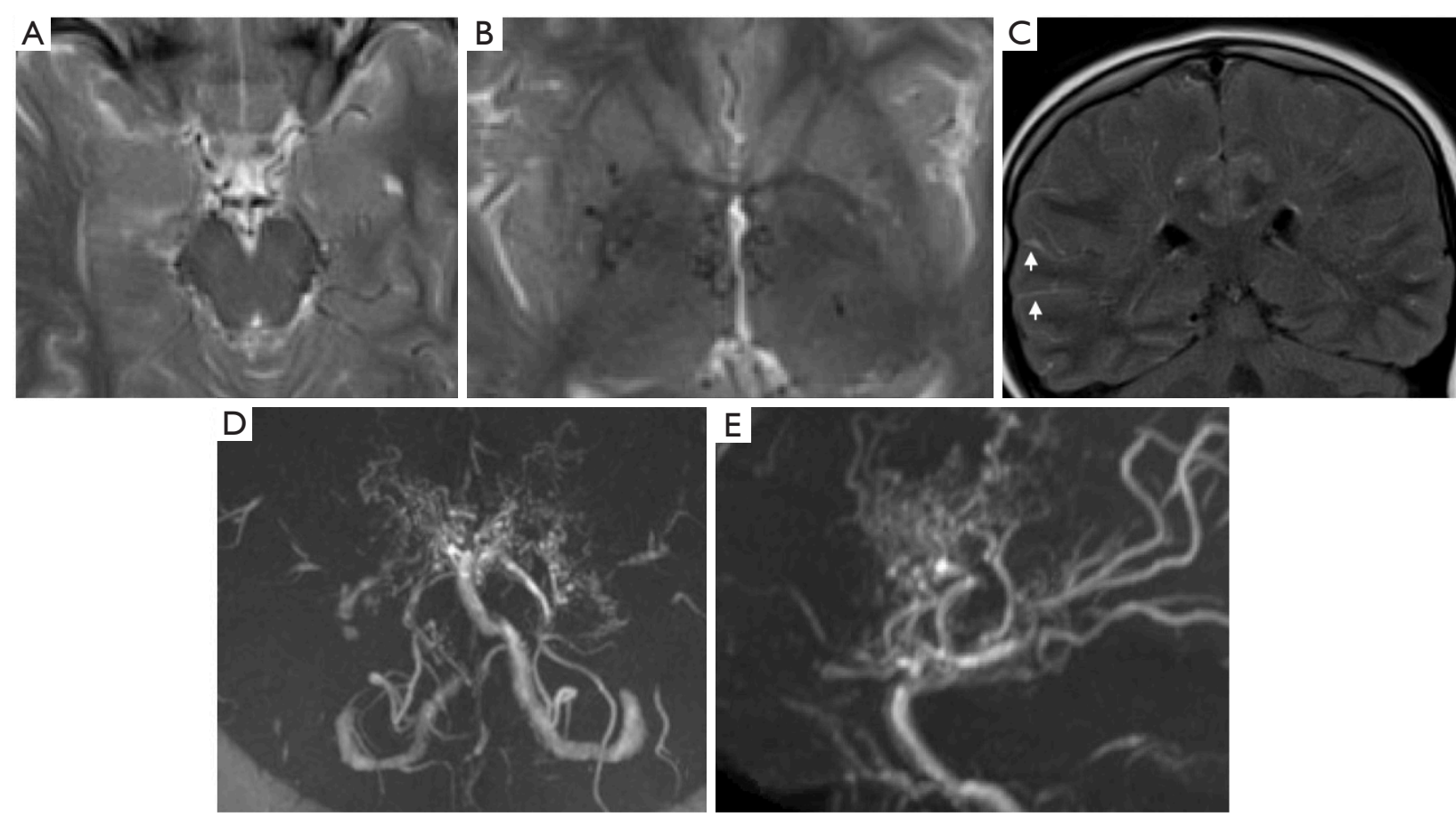

Figure 5 Moyamoya syndrome in a 14 years old girl. Axial T2WI (A and B) demonstrate prominence of flow voids in the suprasellar cistern with significant narrowing of the ICA bilaterally. There are also multiple perforators noted in the basal ganglia and thalami. Coronal FLAIR image (C) shows the characteristic 'ivy sign' (arrows). TOF MR angiogram (D and E: MIP images) demonstrates bilateral distal carotid and A1 stenosis, as well as reduced M1 flow. There are numerous leptomeningeal collaterals. TOF, time of flight.

in particular ASL, is also important in these patients to assess for critical ischaemia and hypoperfusion. Vessel wall imaging may help differentiate moyamoya disease from other vasculopathies if there is diagnostic ambiguity, however, currently there is limited data available in the literature to support its use as a diagnostic tool.

The most common findings described in literature in Moya Moya disease on vessel wall imaging include non-enhancing non remodeling lesions without $\mathrm{T} 2 \mathrm{~W}$ heterogeneity $(17,21)$. When enhancement was present, this was homogenous and concentric (21). This is in contrast with CNS vasculitis where multifocal areas of concentric enhancement may be seen (21). Confirmation of these findings with larger studies is imperative to validate its clinical use in the paediatric population.

If moyamoya is identified on imaging, further evaluation with digital subtraction angiograph (DSA) is recommended (6). DSA can identify transdural collaterals that are critical biomarkers in assessing angiogenic potential (6).

Children with moyamoya syndrome should be referred to a tertiary paediatric neurosurgical centre for consideration of external to internal carotid bypass.

\section{Sickle cell disease}

Sickle cell disease is an independent and important risk factor for strokes, causing both clinical and silent strokes. Dehydration in these children can lead to occlusive crises and stroke. Sickle cell disease is also associated with moyamoya syndrome, chronic small vessel disease, and cerebral fat emboli from bone marrow necrosis (22-24). Children with sickle cell disease may also present with posterior reversible encephalopathy (PRES) and have a higher risk of aneurysms and intracranial haemorrhage $(24,25)$.

\section{Arterial dissection}

This is a common and underdiagnosed cause of stroke in children accounting for $7 \cdot 5-20 \%$ of childhood AIS $(8,26)$. This potentially treatable cause of stroke should be considered with children, and although anterior circulation is more commonly involved in children, posterior circulation stroke can occur (26). There may be a history of minor trauma. Vertebral artery dissection is most commonly demonstrated as the vertebral artery travels in the transverse 

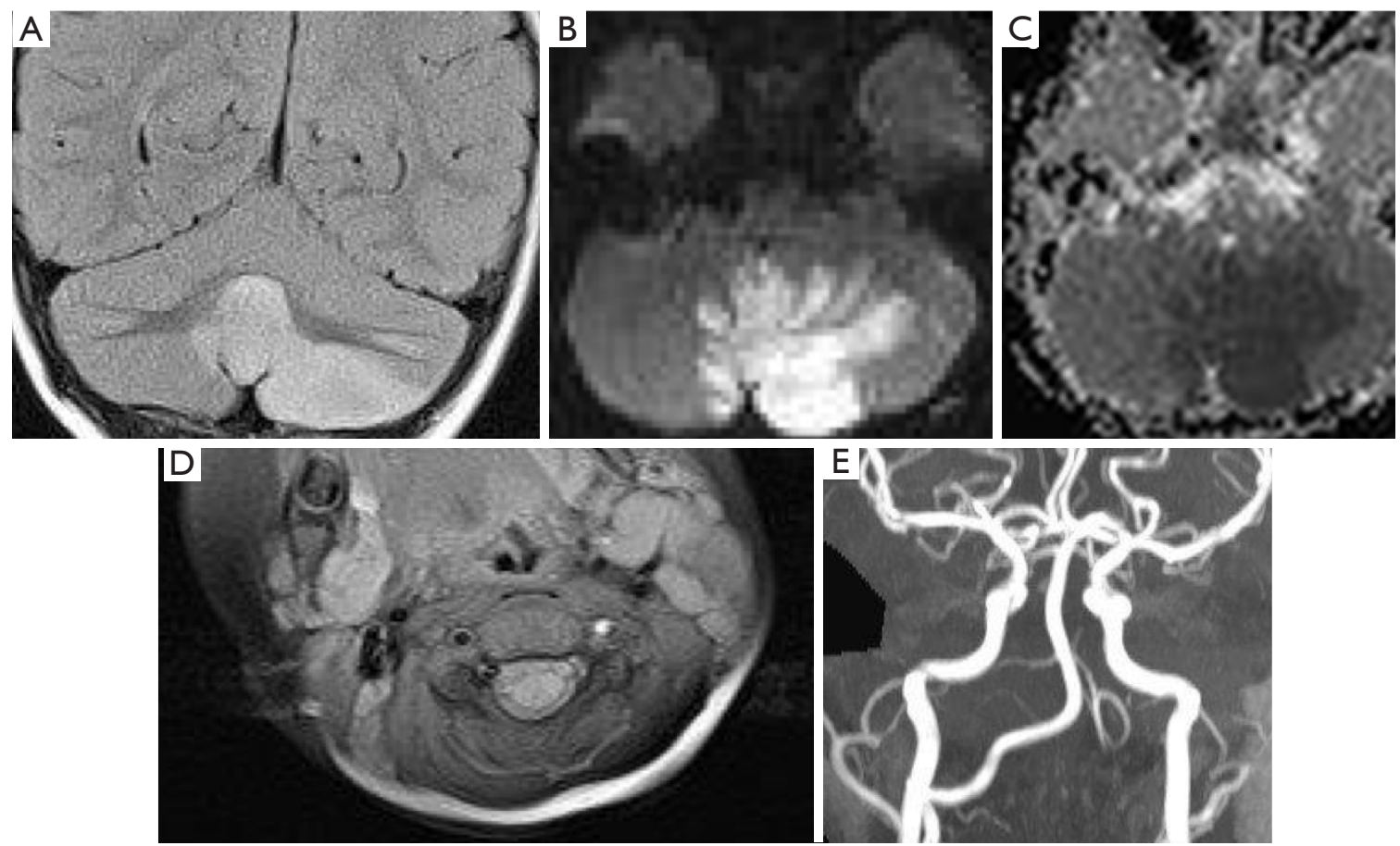

Figure 6 Vertebral artery dissection. Coronal FLAIR image (A) shows signal abnormality within the left cerebellar hemisphere and cerebellar vermis. Diffusion weighted imaging (B: B1000 and C: ADC) show diffusion restriction corresponding to the FLAIR signal abnormality in keeping with acute infarction. Axial T1W fat saturated image (D) shows intrinsic T1W hyperintensity in the upper cervical segment of the left vertebral artery. Time-of-flight MRA (E) shows a corresponding lack of normal flow within the left vertebral artery related to dissection. FLAIR, fluid attenuated inversion recovery; ADC, apparent diffusion co-efficient; MRA, MR angiogram.

foramen (V2) and as it exits the transverse foramen at C2 before entering the foramen magnum (27). Internal carotid dissection is most commonly seen just above the carotid bifurcation (V3) or at the skull base (27). More diffuse involvement may be seen in children with connective tissue disorders, such as, Marfan's, Ehlers Danlos syndrome type IV, Osteogenesis imperfecta type I, autosomal dominant polycystic kidney disease, fibromuscular dysplasia, and Menke's disease.

While catheter angiogram is 'the gold standard investigation', the diagnosis is usually made on CT or MR angiography $(6,27)$. Visualisation of arterial stenosis, an intimal flap or a double lumen in the artery are pathognomonic features of a dissection, however, these are not always present (Figure 6) (27). A tapering arterial occlusion described in literature as a 'string sign' or 'flame sign', aneurysmal dilatation of the artery or an eccentric mural thrombus are less specific signs (27).

\section{CNS vasculitis}

Vasculitis in children can be either isolated within the CNS or secondary to systemic involvement. Recurrent strokes, the presence of encephalopathy, fever or rash, co-existent reno-vascular disease or raised inflammatory markers should all raise the suspicion of a vasculitic process.

Appearances on imaging can be non-specific (Figure 7). Multifocal areas of infarction with involvement of more than one territory should raise the suspicion of vasculitis (28). MRI with contrast may also show leptomeningeal or parenchymal enhancement (28). There may be involvement of more than one vessel and both neck and intracranial vasculature can be involved. The most common pattern in children is unilateral, multifocal, and proximal artery involvement (28). There may also be evidence of previous infarction. Vessel irregularity, focal stenosis followed by dilatation, also referred to as beading and aneurysm formation can be observed on CT or MR angiogram (28). It should be noted that abnormal angiographic findings are predominantly seen in large or medium vessel vasculitis. Therefore, a negative angiogram does not exclude small vessel vasculitis and for diagnosis, correlation with serum 

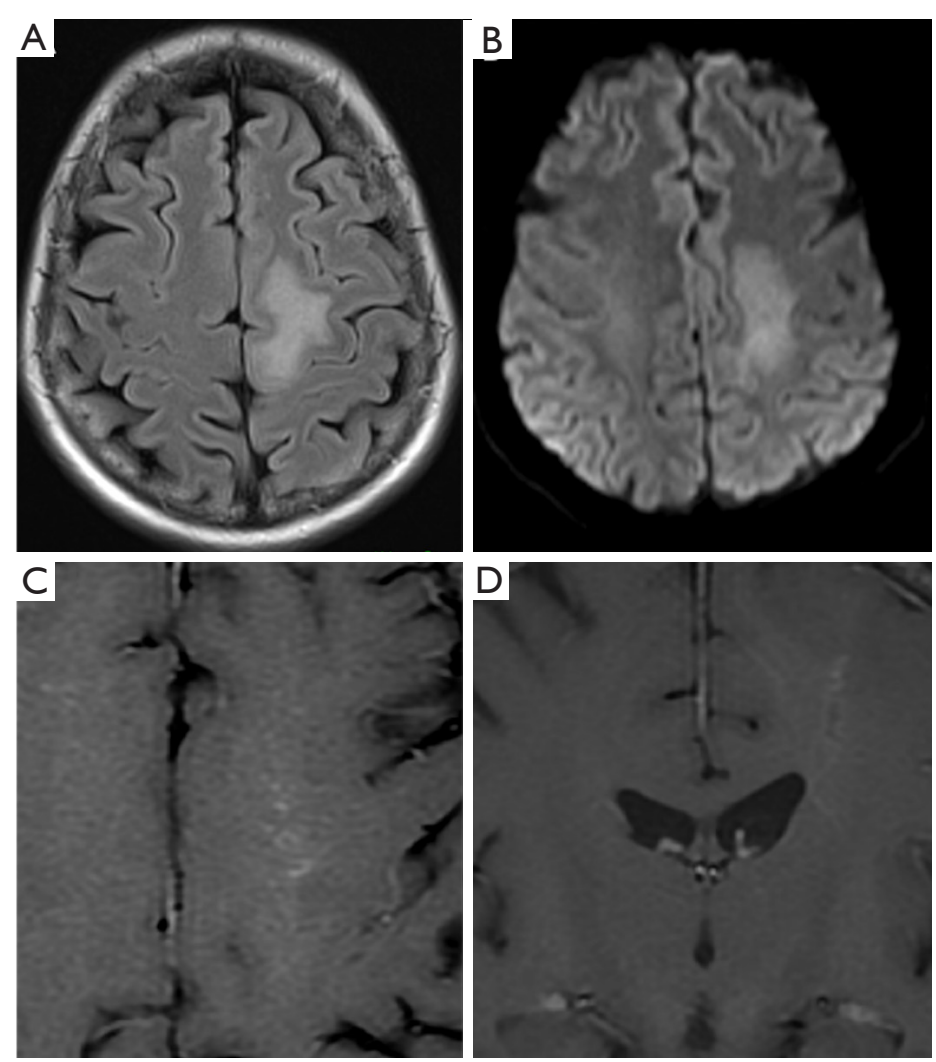

Figure 7 Biopsy proven vasculitis in a 13-year-old child who presented with history of worsening gait and left sided neck pain. Axial T2WI (A) and axial DWI B1000 (B) images demonstrate a hazy signal abnormality within the left centrum semiovale with corresponding mild diffusion restriction. Post contrast axial and coronal T1WI (C and D) show patchy serpiginous enhancement within this region. DWI, diffusion weighted imaging.

markers and consideration for brain biopsy may need to be raised. Vessel wall imaging may provide further information and help differentiate vasculitis from other vascular abnormalities. Pathologically, the presence of inflammatory changes and oedema within the vessel wall is represented as wall thickening, and multifocal concentric arterial wall enhancement (17).

\section{Congenital heart diseases}

Children with underlying heart conditions, particularly cyanotic heart disease are at greater risk of suffering embolic infarcts $(6,9)$. Although the cause of stroke is usually thromboembolic, it is important to note that patients with cardiac disease may have an associated arteriopathy and therefore cerebrovascular imaging in these patients is important $(6,8)$. This risk is increased at the time of bypass surgery. Children without a known heart condition presenting with stroke should also have a cardiac echocardiogram to exclude a patent foramen ovale (6).

\section{Metabolic and genetic risk factors}

There is a growing list of genetic and metabolic disorders associated with ischaemic strokes in childhood $(6,7)$. An example case of ACTA2 mutation is shown (Figure 2). These are summarised in Table 3.

\section{Management and follow up of AIS}

There has been significant research in the last decade in the timing of endovascular treatment in adults with stroke. Unfortunately, there is a lack of evidence for management of hyperacute stroke with endovascular treatment in children and further research is needed to clarify the benefits of invasive treatment (7). Several special considerations need to be discussed and scrutinised including weight limitations for contrast administration, smaller arteries 
Table 7 Recommended imaging protocol in patients with suspected CVST

\begin{tabular}{ll}
\hline Imaging & Recommended imaging protocol \\
\hline MR & $\begin{array}{l}\text { Axial T2WI, sagittal T1WI, DWI, SWI/GRE, and MR } \\
\text { venogram (ideally contrast enhanced T1W sequence, } \\
\\
\text { TOF imaging is more prone to pitfalls and is better } \\
\text { suited for follow up imaging) }\end{array}$ \\
CT & If only CT available: unenhanced CT head and CT \\
& venography should be performed as a first line test \\
\hline
\end{tabular}

CVST, cerebral venous sinus thrombosis; TOF, time of flight.

Table 8 Pitfalls in CT and MR when assessing for CVST

Common pitfalls in imaging interpretation
Beam artefact from the base of skull and applying correct
window levels may mimic or hide transverse sinus thrombosis
Physiological polycythaemia in neonates and polycythaemia in
children may mimic extensive CVST
Acute subdural haemorrhage surrounding the dural venous
sinus and tentorium cerebelli may mimic thrombus on both CT
and MR

CVST, cerebral venous sinus thrombosis.

for cannulation, exposure to radiation at a young age, and introduction of a catheter in an acutely inflamed vessel or chronic arteriopathy. The combination of these issues for consideration makes the use of endovascular treatment in children controversial. The decision to adopt endovascular treatment should be done on a case to case basis (7).

\section{CVST}

\section{Epidemiology and risk factors}

CVST is an uncommon cerebrovascular disease and potentially life threatening in children. The incidence is estimated to be approximately $0.6 / 100,000$ per year (29).

Neonates are at increased risk of poorer prognosis with motor and cognitive impairment as well as epilepsy. Children have a better prognosis with most children making a full recovery. The recurrence rate is $<10 \%$ (29).

The pathophysiology is not fully understood but is thought to be related to one of three mechanisms: stasis or slowing of blood flow, injury or disruption to the blood vessel wall, or alteration in the components of blood affecting coagulation processes leading to clot formation (29).

There are several risk factors identified in children with
CVST with the most common being acute provoking illness $(29,30)$. Acute provoking illnesses may include head and neck infection as well as meningitis. Other risk factors include acute systemic illnesses, underlying chronic diseases, and haematological disorders (29). Important chronic diseases to consider include congenital heart disease, nephrotic syndrome, immunological disorders, anaemia and leukaemia (29).

Clinical presentation is highly variable in CVST and add to the diagnostic dilemma. The typical triad described in literature includes depressed or altered mental state, headache, and vomiting which progress over a period of days (29). Seizures are common particularly in neonates (29). The presence of seizures or coma at presentation signal a poorer prognosis. Depending on the sinus or veins involved, localising signs and symptoms including, sensory or motor deficits, cranial nerve palsies may be present and may help localise the clot.

\section{Diagnostic workup}

CT and MR are often the imaging modalities performed in paediatric patients with suspected acute/subacute cerebral disorders. The recommended imaging protocol is discussed in Table 7.

It is paramount to be familiar with the various normal variants as these can be mistaken for venous sinus thromboses. One such pitfall is arachnoid granulations, which appear as filling defects on CT or MR venograms. Other common pitfalls include asymmetry of the transverse sinuses, sinus bifurcations (common in the superior sagittal sinus) and variations in the anatomy of cortical veins. There are also radiographic pitfalls on CT and MR to be aware of when interpreting venograms (Table 8).

In patients with CVST, an unenhanced CT may be normal in $30-40 \%$ of cases $(31,32)$. Direct signs such as the dense delta sign or cord sign may be observed on plain CT imaging (Figure 8). CT venogram may demonstrate non-opacification of the affected cortical veins or venous sinuses (also known as empty delta sign). Indirect signs include a non-haemorrhagic infarct which does not respect vascular territorial boundaries, or haemorrhage, either intraparenchymal or subarachnoid in location.

MR imaging is also commonly performed in these cases, and the protocol should include axial T2WI, T1WI, DWI, SWI and venogram (which may be time of flight or contrast enhanced). There are also pitfalls to interpretation of MR imaging features. A good understanding of the 

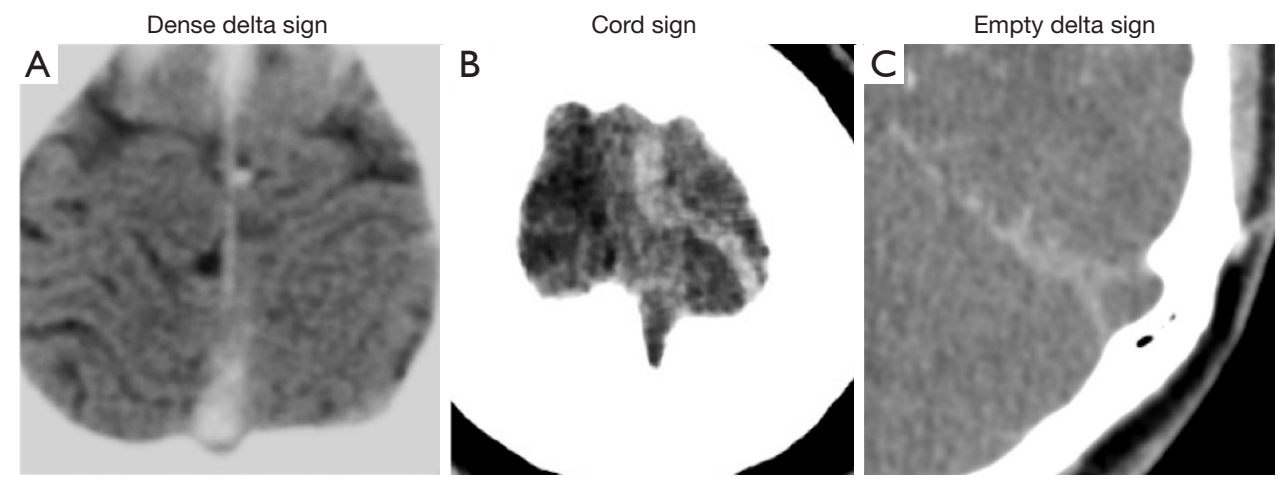

Figure 8 Direct neuroimaging signs of CVST on unenhanced CT (A and B) and contrast enhanced CT venography (C). CVST, cerebral venous sinus thrombosis.

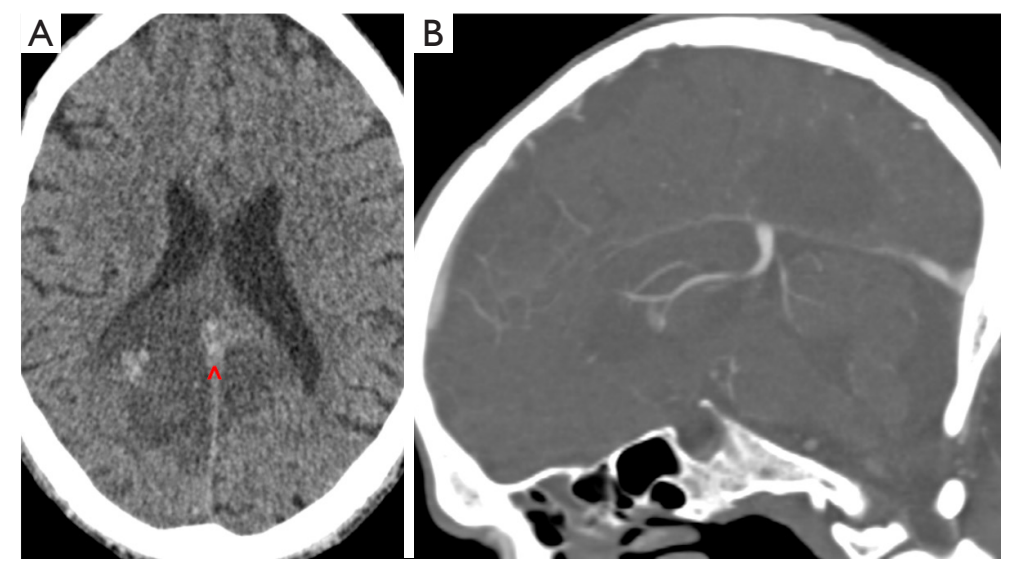

Figure 9 Deep venous sinus thrombosis. Unenhanced CT (A) in an acutely unwell child demonstrated low attenuation changes in the parieto-occipital regions bilaterally, more on the right side, with some areas of associated haemorrhage. This was initially thought to be tumour, and post contrast imaging was performed. However, on further review, subtle hyperdensity was seen along the straight sinus on the plain CT $(\wedge)$. Straight sinus thrombosis was then confirmed by a CT venogram $(B)$.

MR appearances of blood and the degradation products is essential in recognising CVST. Non-contrast T1W sequence is often difficult to interpret in these cases as slow flow can also present with hyperintensity within the sinus. SWI can be extremely useful in the diagnosis of CVST and was found to be positive in all 7 patients in one case series (33). It is useful in recognising acute and subacute thrombus and is particularly helpful in cases with cortical vein thrombosis which can be difficult to visualise on the other sequences. Another benefit of SWI is recognition of petechial parenchymal haemorrhage secondary to venous infarction which may be overlooked on the other sequences. DWI is performed to diagnose any associated venous infarction.
Time of flight MR venogram is sensitive at visualising slow or static blood flow, however, it is therefore susceptible to flow related artefacts. Using contrast enhanced MR venogram can be more useful as it reduces the flow related artefacts but requires the use of gadolinium. A case of deep venous sinus thrombosis with parenchymal haemorrhage has been shown (Figure 9).

\section{Management and follow up of CVST}

Although there is limited research evaluating the risks and benefits of treatment in CVST in children, there are published guidelines which offer some recommendations $(29,34)$. The British Committee for 
Table 9 Risk factors associated with haemorrhagic stroke

\begin{tabular}{ll}
\hline Risk category & Risk factors/conditions \\
\hline Vascular disorders & Arteriovenous malformations \\
& Cavernous malformations \\
& Cerebral aneurysms \\
& Moyamoya syndrome \\
Clotting disorders & Severe platelet disorders \\
& Inherited bleeding disorders \\
& Anticoagulation \\
Sickle cell disease & Severe vitamin K deficiency \\
Illicit drug use & Cocaine \\
Demographic factors & Amphetamines \\
& Black ethnicity years \\
& Male gender
\end{tabular}

Table 10 Recommended imaging protocol for HS

\begin{tabular}{ll}
\hline Imaging & Recommended imaging protocol \\
\hline CT & $\begin{array}{l}\text { Unenhanced CT head should be performed as } \\
\text { a first line investigation in the acute setting. CT } \\
\text { angiogram should be added to evaluate for cause of } \\
\text { haemorrhage }\end{array}$ \\
MR & $\begin{array}{l}\text { If cause not clear on CT, MR imaging can be } \\
\text { performed. Axial T2WI, sagittal T1WI, axial or coronal }\end{array}$ \\
& FLAIR, DWI, SWI/GRE, and TOF MR angiogram
\end{tabular}

FLAIR, fluid attenuated inversion recovery; DWI, diffusion weighted imaging; SWI, susceptibility weighted imaging; GRE, gradient echo; TOF, time of flight.

Standards in Haematology recommend children with CVST and no intracranial haemorrhage should receive anticoagulation therapy which should be continued for 3 months in cases of reversible provoking illness (such as infection) and 6 months in the absence of provoking illness (34). Longer periods of anticoagulation may also be considered if there is persistent symptomatic venous outflow obstruction, long lasting or genetic risk factor (34). Anticoagulation may also be considered in patients with minimal or asymptomatic intracranial haemorrhage (34).

\section{Haemorrhagic stroke}

\section{Epidemiology and risk factors}

Haemorrhagic stroke is defined as non-traumatic intracranial haemorrhage which can be subdivided into intracerebral, subarachnoid and intraventricular haemorrhage. The incidence of haemorrhagic stroke lies between 1-3.2 per 100,000 per year and it accounts for approximately $50 \%$ of all childhood strokes $(2-4,35,36)$.

The risk factors associated with haemorrhagic stroke vary from AIS and are outlined in Table 9. The most common causes include arteriovenous malformations (AVMs), cavernous malformations (CM) and aneurysms. Patients with sickle cell disease have a high cumulative incidence of bleeding of $21 \%$ at age 40 and this rises to $41 \%$ by age 60 (37). There is also a higher risk of haemorrhagic transformation with ischaemic stroke in these patients (37). Acute focal neurological deficit, seizure and headache were the three most common clinical presentations in children with haemorrhagic stroke (7). As these are also the most common presentations in AIS, it is not possible to clinically distinguish between AIS and HS. There is a risk of developing hydrocephalus early in these patients either secondary to intraventricular haemorrhage or due to compression and mass effect from intracerebral haemorrhage.

\section{Diagnostic workup}

Children identified with haemorrhagic stroke should undergo discussion at a neurovascular multidisciplinary team meeting to determine the timing and modality of further investigations and plan for treatment. Imaging is usually in the form of CTA or MRA. In addition, haematological investigations including coagulation screen, full blood count and haemoglobinopathy screen is recommended. If the child is also known to have sickle cell disease, transcranial Doppler ultrasound and an extended blood group phenotype are recommended. An imaging protocol is suggested in Table 10 .

\section{Background, imaging and management of specific conditions}

\section{High flow vascular malformations}

AVMs are the cause of HS in $39 \%$ of cases (36).

CT/CTA demonstrate tortuous serpentine vessels with 

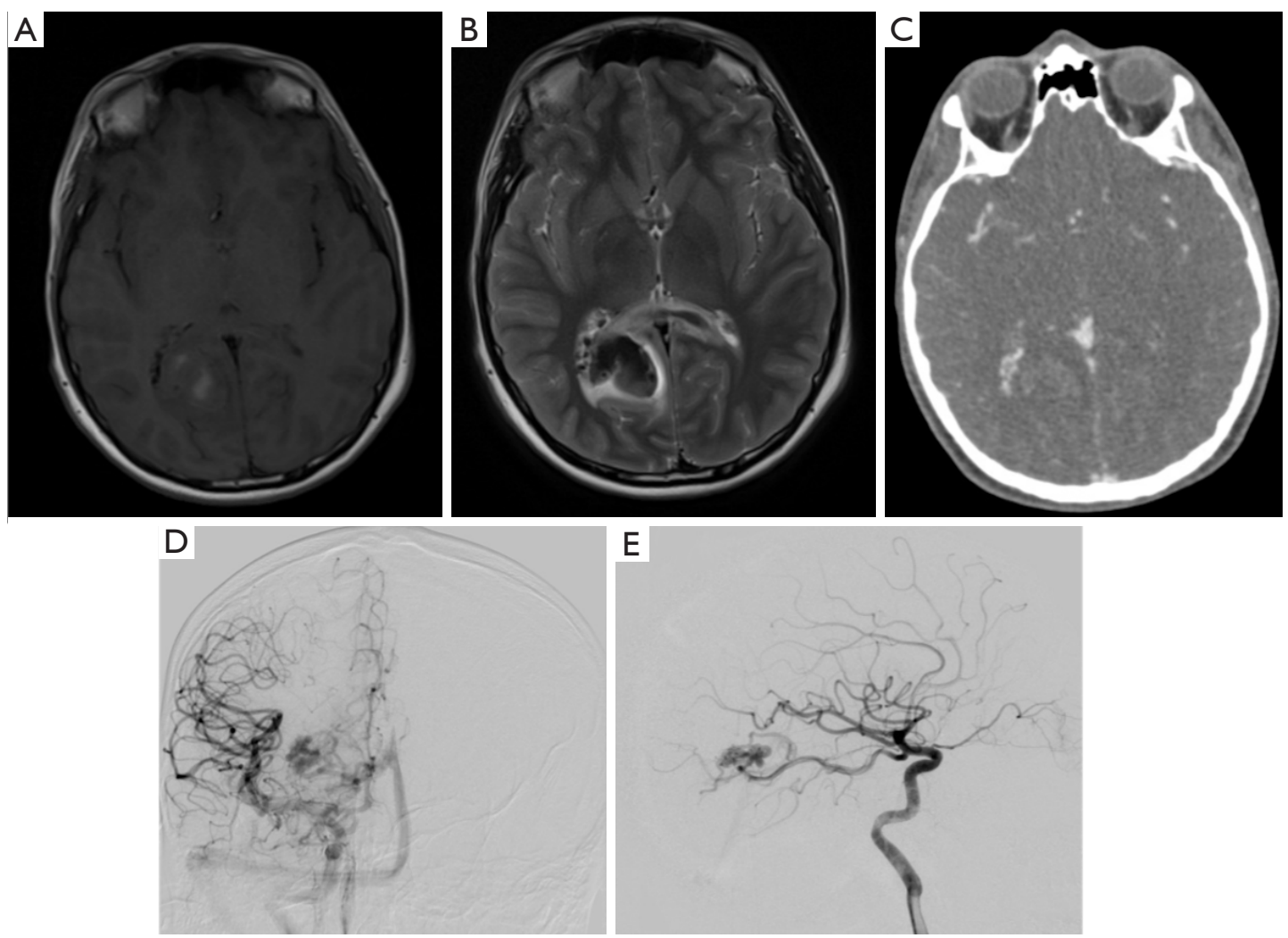

Figure 10 Haemorrhagic stroke with an AVM. A 15-year-old male presented with acute onset headache and vomiting. Axial T1W and T2W images (A and B respectively) shows a right occipital lobar haemorrhage with localised mass effect and signal change extending into the splenium of the corpus callosum. The T2W axial image also demonstrates multiple abnormal flow voids at the anterolateral aspect of the haematoma. Subsequent CT angiogram (C) shows a cluster of vessels corresponding to the abnormal flow voids seen on MR in keeping with a vascular malformation. Digital subtraction angiography (DSA) coronal and sagittal images (D and E) confirms a right occipital AVM with a single arterial feeder from the PCA and venous drainage via the deep venous system. AVM, arteriovenous malformation.

areas of calcification, an arterial feeder, central vascular nidus and draining veins. MR imaging show characteristic serpiginous signal voids of the dilated vessels on $\mathrm{T} 2$, areas of haemosiderin staining on gradient echo sequences, and $\mathrm{T} 1 \mathrm{~W}$ hyperintensity associated with areas of haemorrhage (Figure 10).

Arteriovenous fistulas differ from AVMs as they lack a central nidus between the communicating artery and vein. Although rare, these account for $4 \%$ of paediatric cerebral vascular malformations (38).

\section{CM}

Paediatric CM accounts for a quarter of the total of all CMs with an estimated incidence of $0.37-0.53 \%$ among the paediatric population (39). CMs are found to be the cause in $11 \%$ of cases (36). CM consist of dilated sinusoidal vessels lined by endothelium devoid of smooth muscle and without intervening neural parenchyma.

On imaging, appearances can be variable depending on the stage of haemorrhage. Classically on CT/CTA, a CM is round and high in attenuation relating to calcification or haemorrhage. The degree of surrounding low attenuation or oedema and the associated mass effect is dependent on the presence of recent haemorrhage. MR shows a characteristic 'popcorn' appearance with a haemosiderin ring which blooms on SWI. SWI is extremely useful in evaluating for further smaller lesions which are not clearly visualised on the conventional sequences and may suggest an underlying syndrome such as multiple CM syndrome. Depending on the stage of haemorrhage, T1/T2W signal will vary. Surrounding oedema is clearly visualised on $\mathrm{T} 2 \mathrm{~W}$ and FLAIR sequences and this coupled with a change in size or morphology can be indicative of recent haemorrhage.

Management should be discussed in an MDT meeting 
Table 11 Recommended follow up protocol in patients with AVMs and aneurysms. Adapted from RCPCH guidelines 2017 (7)

\begin{tabular}{ll}
\hline Treatment & Follow up \\
\hline AVMs & \\
Endovascular treatment & Catheter angiography at 3-6 months after procedure \\
Surgical resection & MR + MRA and catheter angiogram at 3-6 months post surgery \\
Stereotactic radiosurgery (SRS) & MR and MRA at 2 years following treatment. If no residuum, confirm with catheter angiogram \\
Aneurysms & \\
Endovascular treatment & MR and MRA every 3-6 months for 2 years and further follow-up determined on a case-by-case basis \\
Surgical management & A single Catheter angiogram demonstrating obliteration of the aneurysm at 3-6 months \\
\hline
\end{tabular}

AVM, arteriovenous malformation; MRA, MR angiogram.

and options include observation or surgical management. Surgery is considered in children with acute haemorrhage or focal neurological deficits. This is particularly true for infratentorial lesions.

\section{Aneurysms}

Aneurysms, accounting for $9 \%$ of HS in children, are rare and pathologically different to those in adults (36). They tend to be larger in size, with a higher incidence of giant aneurysms and are more likely to be associated with other congenital diseases.

Management should be discussed within an MDT meeting, and options include both endovascular coiling and surgical clipping. Due to the incidence of re-bleeds following endovascular treatment, surgical clipping may be more appropriate in the paediatric population.

\section{Follow up imaging in $\mathrm{HS}$}

A follow up protocol in patients with AVMs and aneurysms has been recommended by the RCPCH guidelines 2017 and comprises of MR + MRA and/or catheter angiography (Table 11). Due to the lack of evidence, this is based upon the Delphi panel consensus meeting discussions and there is some variation in protocols between centres.

\section{Conclusions}

Childhood stroke is a well-recognised cause of significant morbidity and mortality. Despite recent advances in our understanding of strokes in general, numerous challenges continue to result in delays in diagnosing and treating childhood strokes. There is a growing need for streamlining diagnostic protocols to allow for efficient management.

Childhood stroke also presents more variably than adult strokes and is often missed initially. AIS remains the most common cause and the risk factors in children vary significantly from adult ischaemic strokes, with arteriopathy being the most common underlying factor. Urgent imaging in the form of CT or MR brain should be carried out within an hour and a thorough search for underlying causes should be performed. The role of endovascular intervention is not as well established in children, as it is in adult strokes. Cerebral venous sinus thromboses in children are much less frequent, and are often due to local or systemic illness. Haemorrhagic infarcts are also rare and usually associated with an underlying vascular malformation. Challenges exist in the diagnostic evaluation and management of haemorrhagic stroke and CVST with lack of national or international consensus. Further research is required in these fields.

This practical guide aimed to provide an overview of the risk factors, diagnostic evaluation, imaging protocols and management of paediatric stroke stratified according to AIS, HS and CVST.

\section{Acknowledgments}

Our thanks to Dr. Julija Pavaine (Consultant Paediatric Neuroradiologist, Royal Manchester Childrens' Hospital), and to our neuroradiology colleagues at GOSH (Great Ormond Street Hospital) for some of the cases. Funding: None.

\section{Footnote}

Provenance and Peer Review: This article was commissioned by 
the Guest Editor (Felice D'Arco) for the series "Pediatric Neuroradiology for Trainees and Fellows: An Updated Practical Guide" published in Translational Pediatrics. The article has undergone external peer review.

Conflicts of Interest: Both authors have completed the ICMJE uniform disclosure form (available at http:// dx.doi.org/10.21037/tp-20-205). The series "Pediatric Neuroradiology for Trainees and Fellows: An Updated Practical Guide" was commissioned by the editorial office without any funding or sponsorship. The authors have no other conflicts of interest to declare.

Ethical Statement: The authors are accountable for all aspects of the work in ensuring that questions related to the accuracy or integrity of any part of the work are appropriately investigated and resolved.

Open Access Statement: This is an Open Access article distributed in accordance with the Creative Commons Attribution-NonCommercial-NoDerivs 4.0 International License (CC BY-NC-ND 4.0), which permits the noncommercial replication and distribution of the article with the strict proviso that no changes or edits are made and the original work is properly cited (including links to both the formal publication through the relevant DOI and the license). See: https://creativecommons.org/licenses/by-nc-nd/4.0/.

\section{References}

1. WHO MONICA Project Investigators. The World Health Organization MONICA Project (Monitoring trends and determinants in cardiovascular disease). J Clin Epidemiol 1988;41:105-14.

2. Lehman LL, Khoury JC, Taylor JM, et al. Pediatric Stroke Rates Over 17 Years:Report From a Population-Based Study. J Child Neurol 2018;33:463-7.

3. Fullerton HJ, Wu YW, Zhao S, et al. Risk of stroke in children: ethnic and gender disparities. Neurology 2003;61:189-94.

4. Chiang KL, Cheng CY. Epidemiology, risk factors and characteristics of pediatric stroke: a nationwide populationbased study. QJM 2018;111:445-54.

5. Benjamin EJ, Muntner P, Alonso A, et al. Heart Disease and Stroke Statistics-2019 Update:A Report From the American Heart Association [published correction appears in Circulation 2020 Jan 14;141(2):e33]. Circulation 2019;139:e56-e528.
6. Ferriero DM, Fullerton HJ, Bernard TJ, et al. Management of Stroke in Neonates and Children:A Scientific Statement From the American Heart Association/American Stroke Association. Stroke 2019;50:e51-96.

7. RCPCH. Stroke in childhood - clinical guideline for diagnosis, management and rehabilitation 2017. Available online: https://www.rcpch.ac.uk/sites/default/ files/2019-04/Stroke\%20guideline\%2008.04.19.pdf. [Accessed on 20th July 2020]

8. Amlie-Lefond C, Bernard TJ, Sébire G, et al. Predictors of cerebral arteriopathy in children with arterial ischemic stroke: results of the International Pediatric Stroke Study. Circulation 2009;119:1417-23.

9. Ganesan V, Prengler M, McShane MA, et al. Investigation of risk factors in children with arterial ischemic stroke. Ann Neurol 2003;53:167-73.

10. Mallick AA, Ganesan V, Kirkham FJ, et al. Childhood arterial ischaemic stroke incidence, presenting features, and risk factors: a prospective population-based study. Lancet Neurol 2014;13:35-43.

11. Mallick AA, Ganesan V, Kirkham FJ, et al. Diagnostic delays in paediatric stroke. J Neurol Neurosurg Psychiatry 2015;86:917-21.

12. Ladner TR, Mahdi J, Gindville MC, et al. Pediatric Acute Stroke Protocol Activation in a Children's Hospital Emergency Department. Stroke 2015;46:2328-31.

13. Rafay MF, Pontigon AM, Chiang J, et al. Delay to diagnosis in acute pediatric arterial ischemic stroke. Stroke 2009;40:58-64.

14. Chen J, Licht DJ, Smith SE, et al. Arterial spin labeling perfusion MRI in pediatric arterial ischemic stroke: initial experiences. J Magn Reson Imaging 2009;29:282-90.

15. Proisy M, Bruneau B, Rozel C, et al. Arterial spin labeling in clinical pediatric imaging. Diagn Interv Imaging 2016;97:151-8.

16. Donahue MJ, Dlamini N, Bhatia A, et al. Neuroimaging Advances in Pediatric Stroke. Stroke 2019;50:240-8.

17. Young CC, Bonow RH, Barros G, et al. Magnetic resonance vessel wall imaging in cerebrovascular diseases. Neurosurg Focus 2019;47:E4.

18. Dlamini N, Yau I, Muthusami P, et al. Arterial Wall Imaging in Pediatric Stroke. Stroke 2018;49:891-8.

19. Centeno MAR, Buompadre MC, Requejo F, et al. Arterial Ischemic Stroke in Childhood. Curr Treat Options Peds 2019;5:380-96.

20. Braun KP, Bulder MM, Chabrier S, et al. The course and outcome of unilateral intracranial arteriopathy in 79 children with ischaemic stroke. Brain 2009;132:544-57. 
21. Mossa-Basha M, de Havenon A, Becker KJ, et al. Added Value of Vessel Wall Magnetic Resonance Imaging in the Differentiation of Moyamoya Vasculopathies in a NonAsian Cohort. Stroke 2016;47:1782-8.

22. Saito N, Nadgir RN, Flower EN, et al. Clinical and radiologic manifestations of sickle cell disease in the head and neck. Radiographics 2010;30:1021-34.

23. Dhakal LP, Bourgeois K, Barrett KM, et al. The "starfield" pattern of cerebral fat embolism from bone marrow necrosis in sickle cell crisis. Neurohospitalist 2015;5:74-6.

24. Thust SC, Burke C, Siddiqui A. Neuroimaging findings in sickle cell disease. Br J Radiol 2014;87:20130699.

25. Lonergan GJ, Cline DB, Abbondanzo SL. Sickle cell anemia. Radiographics 2001;21:971-94.

26. Mirsky DM, Beslow LA, Amlie-Lefond C, et al. International Paediatric Stroke Study Neuroimaging Consortium and the Paediatric Stroke Neuroimaging Consortium. Pathways for Neuroimaging of Childhood Stroke. Pediatr Neurol 2017;69:11-23.

27. Stence NV, Fenton LZ, Goldenberg NA, et al. Craniocervical arterial dissection in children: diagnosis and treatment. Curr Treat Options Neurol 2011;13:636-48.

28. Aviv RI, Benseler SM, Silverman ED, et al. MR imaging and angiography of primary CNS vasculitis of childhood. AJNR Am J Neuroradiol 2006;27:192-9.

29. Ichord R. Cerebral Sinovenous Thrombosis. Front Pediatr 2017;5:163.

30. Ichord RN, Benedict SL, Chan AK, et al. Paediatric

Cite this article as: Sinha R, Ramji S. Neurovascular disorders in children: an updated practical guide. Transl Pediatr 2021;10(4):1100-1116. doi: 10.21037/tp-20-205 cerebral sinovenous thrombosis: findings of the International Paediatric Stroke Study. Arch Dis Child 2015;100:174-9.

31. Dlamini N, Billinghurst L, Kirkham FJ. Cerebral venous sinus (sinovenous) thrombosis in children. Neurosurg Clin N Am 2010;21:511-27.

32. Roland T, Jacobs J, Rappaport A, et al. Unenhanced brain CT is useful to decide on further imaging in suspected venous sinus thrombosis. Clin Radiol 2010;65:34-9.

33. Singh R, Cope WP, Zhou Z, et al. Isolated cortical vein thrombosis:case series. J Neurosurg 2015;123:427-33.

34. Chalmers E, Ganesen V, Liesner R, et al. Guideline on the investigation, management and prevention of venous thrombosis in children. Br J Haematol 2011;154:196-207.

35. Jordan LC, Johnston SC, Wu YW, et al. The importance of cerebral aneurysms in childhood hemorrhagic stroke:a population-based study. Stroke 2009;40:400-5.

36. Jordan LC, Hillis AE. Hemorrhagic stroke in children. Pediatr Neurol 2007;36:73-80.

37. Hariharan N, Brunson A, Mahajan A, et al. Bleeding in patients with sickle cell disease: a population-based study. Blood Adv 2020;4:793-802.

38. Cooke D, Tatum J, Farid H, et al. Transvenous embolization of a pediatric pial arteriovenous fistula. $\mathrm{J}$ Neurointerv Surg 2012;4:e14.

39. Mottolese C, Hermier M, Stan H, et al. Central nervous system cavernomas in the pediatric age group. Neurosurg Rev 2001;24:55-71. 